\title{
Habilidades sociais e desempenho acadêmico: um estudo comparativo entre os cursos da área de saúde e humanas e cursos de ciências exatas e sociais aplicadas
}

\author{
Thaís Cristina Gutstein Nazar \\ Michele Quaglioto Tartari \\ Ana Caroline Grassi Vanazi \\ Andreia Belusso
}

\begin{abstract}
Resumo: Considerando a importância das Habilidades Sociais no desenvolvimento acadêmico e profissional dos estudantes, esta pesquisa tem por objetivo verificar a existência de relações entre Habilidades Sociais e desempenho acadêmico. Este estudo caracteriza-se por uma pesquisa descritiva correlacional quantitativa, na qual participaram 628 estudantes universitários das áreas de saúde e humanas e exatas e sociais aplicadas que responderam ao Inventário de Habilidades Sociais - IHS (Del Prette \& Del Prette, 2014). Para o tratamento dos dados, foi utilizado o Statistical Package for the Social Sciences (IBM SPSS) 21.0 para Windows, valendo-se de testes correlacionais. Foi possível verificar a correlação entre Habilidades Sociais e desempenho acadêmico nos cursos de saúde e humanas, em contrapartida, não há correlação estatisticamente significativa entre ambos nos cursos das áreas de exatas e sociais aplicadas. Encontra-se respaldo teórico nestas verificações, sendo possível analisá-las neste artigo.
\end{abstract}

Palavras-chaves: Habilidades sociais; Desempenho acadêmico; Universitários.

\section{Social skills and academic performance: a comparative study between health and human curses and exact and social applied areas}

\begin{abstract}
Considering social skills relevance for the academic and professional students development, this study aims to verify the existence of relations between the variables Social Skills and Academic Performance analyzing the factors that compose them to the university environment. This study is characterized by a quantitative correlational descriptive research in which 628 university students of the health and human and applied social and exact areas participated, who answered the Social Skills Inventory IHS (Del Prette \& Del Prette, 2014). For the data processing by using the Statistical Package for the Social Sciences (IBM SPSS) 21.0 for Windows. It was possible to verify the correlation between Social Skills and Academic Performance in the health and human courses, in contrast there is no statistically significant correlation between both in the courses of the exact and social applied areas. There is theoretical support for these verifications and it is possible to analyze it in this article.
\end{abstract}

Keywords: Social skills; Academic performance; University students.

\section{Introdução}

Nos últimos anos, o número de indivíduos que ingressaram no contexto acadêmico cresceu consideravelmente. Esse aumento impactou em mudanças no cenário educacional trazendo a necessidade de se pensar sobre as variáveis que compõem este enquadre, 
dessa forma tem crescido o interesse de vários pesquisadores sobre fatores que envolvem o contexto do ensino superior, entre eles o público acadêmico e o seu desempenho na graduação. (Rodrigues, Resende, Miranda \& Pereira 2016; Miranda, Lemos, Oliveira \& Ferreira 2015; Fagundes, Luce \& Espinar, 2014).

A preocupação com os resultados do desempenho acadêmico é importante por que, ao entender como os alunos aprendem e o que influencia esse processo, é possível pensar estratégias e manejar contingências de maneira a otimizar o processo da aprendizagem (Skinner, 1904/1972). Da mesma forma segundo Mamede, Marques, Rogers e Miranda (2015), tal entendimento possibilita inclusive se pensar em melhorias nas políticas públicas voltadas ao ensino.

O desempenho acadêmico pode ser influenciado por vários fatores, sendo assim, complexo e multideterminado. Neste sentido, tem sido estudado em seus aspectos teóricos e metodológicos e dentre as variáveis pesquisadas apresentadas na literatura disponível encontram-se características socioeconômicas, demográficas, institucionais e pedagógicas, estudantes que trabalham, grau de escolaridade dos pais, modalidade de ingresso no ensino superior (Rocha, Leles \& Queiroz, 2018) história educacional, idade, renda familiar, gênero (Junior \& Amorim, 2014) e estilos de aprendizagem (Santos, Cunha \& Hein, 2017).

Mamede et al. (2015) versam sobre fatores internos e externos e variáveis psicológicas e comportamentais, corpo discente e as próprias instituições enquanto influenciadores no desempenho acadêmico. Silva (2015) elenca também a inteligência, a expectativa e as Habilidades Sociais como variáveis que atuam de maneira favorável ou desfavorável ao desempenho acadêmico. No entanto segundo Miranda et al. (2015) os estudos amiúde focam-se em determinados aspectos, concentram-se em questões socioeconômicas e demográficas e as questões comportamentais são pouco exploradas.

Partindo-se do referencial teórico das Habilidades Sociais, entende-se que estas, quando existentes no repertório comportamental do indivíduo funcionam como fator de proteção, pois favorecem a adaptação do aluno ao contexto acadêmico e servem direta ou indiretamente como facilitadores de um bom desempenho (Elias \& Amaral, 2016), cabe, portanto investigar-se a relação entre Habilidades Sociais e desempenho acadêmico, sendo este o objetivo deste estudo.

O estudo das Habilidades Sociais é um campo recente, está atrelado aos movimentos de treinamento em Assertividade nos Estados Unidos e o Treinamento de Habilidades Sociais na Inglaterra decorrentes dos estudos de Argyle (Del Prette \& Del Prette, 2017; Ferreira, Oliveira \& Vandenberghe, 2014). De acordo com estes autores, os estudos vêm evoluindo na criação de procedimentos e estratégias para estruturar programas de treinamento nesta área.

No Brasil, Habilidades Sociais tem sido foco importante de muitos pesquisadores. Dentre eles, destacam-se Del Prette e Del Prette (2011, p. 31), que conceituam as Habilidades Sociais referindo-se "à existência de diferentes classes de comportamentos sociais no repertório do indivíduo para lidar de maneira adequada com as demandas das situações interpessoais". São compostas por classes de respostas de comportamentos públicos, verbais e não verbais e comportamentos privados, exigidos nos contextos sociais, são classificadas em: habilidades de civilidade, comunicação, assertivas (direito 
e cidadania), empáticas, de trabalho e de sentimento positivo (Del Prette \& Del Prette, 2011) podendo variar de acordo com a situação social, contexto e cultura em que o sujeito está inserido (Caballo, 1955/2016).

Quando o processo de aquisição ocorre de forma eficaz, o indivíduo é considerado socialmente competente, neste sentido as Habilidades Sociais contribuem para que os relacionamentos e as interações sociais ocorram de forma satisfatória para o sujeito e para o grupo. (Soares, Buscacio, Fernandes, Medeiros \& Monteiro, 2017; Del Prette \& Del Prette, 2011). Porém, nem todos conseguem desenvolver ou adquirir elementos comportamentais necessários para as interações sociais, havendo, portanto, uma diferenciação entre sujeito hábil e não hábil (Caballo, 1995/2016).

O déficit em Habilidades Sociais pode ser identificado por meio de observação, aplicação de questionários e inventários, dentre eles podemos destacar o uso dos inventários de autorrelato como o Inventário de Habilidades Sociais - IHS-Del-Prette (Del Prette \& Del Prette, 2014) que afere "o repertório de habilidades sociais usualmente requerido em uma amostra de situações interpessoais e cotidianas" (Del Prette \& Del Prette, 2014, p. 15).

É possível afirmar que as Habilidades Sociais funcionam como fator de proteção, segundo Bolsoni-Silva e Carrara (2010), na medida em que se tornam concorrentes a comportamentos não assertivos relacionados ao desempenho acadêmico. Elias e Amaral (2016) encontraram nos estudos para realização da sua pesquisa, indicações de que indivíduos com baixa competência social (compreendidos por problemas de interação e de comportamento) têm isso refletido em seu desempenho no ambiente acadêmico de modo negativo.

Esse resultado corrobora com Soares et al. (2017) que, em seu estudo, apontam “que o comportamento em sala de aula, apresentado pelos alunos socialmente competentes favorece a capacidade de se posicionar de maneira adequada e coerente". Além disso, Soares et al. (2017) citam que alunos que não apresentavam um repertório de Habilidades Sociais adequado vivenciavam com mais frequência situações de estresse, refletindo no prejuízo das relações e rendimento.

AAnálise do Comportamento, cujas bases filosóficas advêm do Behaviorismo Radical (Moreira \& Medeiros, 2007), defende que a aquisição do repertório comportamental do indivíduo é selecionado pelos níveis filogenético, ontogenético e cultural, (Skinner, 1904/1972). Os estudos sobre Habilidades Sociais compreendem-as como classes de comportamento adquiridos, principalmente no que se refere ao comportamento operante, sendo que esta abordagem da Psicologia contribui na constituição da teoria e aplicação do Treinamento em Habilidades Sociais - THS que se apresenta como uma das possibilidades de colaborar na aquisição de Habilidades Sociais (Del Prette \& Del Prette, 2017).

Diante da relevância do estudo acerca do campo de Habilidades Sociais no contexto universitário, indaga-se se o desempenho acadêmico estaria atrelado ao repertório de Habilidades Sociais dos estudantes. Questiona-se também se existem diferenças nas associações entre o desempenho acadêmico e Habilidades Sociais nas áreas do conhecimento pesquisadas. Assim, esse estudo propôs-se a comparar os resultados encontrados nas correlações realizadas entre os cursos da área da saúde e humanas e os cursos das áreas de exatas e sociais aplicadas. 


\section{Método}

O presente estudo caracteriza-se por uma pesquisa descritiva correlacional quantitativa. Tendo em vista que se interessa "em descrever a maneira pela qual os eventos estão sistematicamente relacionados uns aos outros" (Cozby, 2012, p. 21). Os resultados encontrados são expressos por meio de representação numérica, o que o classifica como um estudo quantitativo (Cozby, 2012). A interpretação e descrição foram realizadas por meio de testes de correlação de Spearman como análise de coeficiente por se tratar de uma amostra não paramétrica.

Este estudo foi realizado nos anos de 2017 e 2018 como parte de um projeto abrangente vinculado ao PIC - Programa de Iniciação Científica, de uma universidade privada da cidade de Francisco Beltrão, estado do Paraná. A seleção da amostra deu-se por conveniência pelo critério acessibilidade ao público alvo da pesquisa, neste caso, universitários. Os participantes foram selecionados a partir dos critérios do aceite em participar, assinatura do TCLE, estar matriculado nas turmas de $2^{\circ}$ ou $3^{\circ}$ ano de cada curso, com idade superior a 18 anos. A coleta de dados foi realizada por meio de questionários e inventários, aplicados na sala de aula de cada curso e série, coletivamente.

Seguiram-se os padrões éticos de pesquisa com seres humanos, o qual foi aprovado pelo Comitê de Ética em Pesquisa da UNIPAR - PARECER No 2.052.864/2017; 2.222.570/2018 e pautou-se nos princípios e normas estabelecidos na Resolução 466/2012. Após o aceite da instituição de ensino participante e da organização para as coletas de dados com as respectivas coordenações dos cursos, foi realizado um rapport explicativo sobre a pesquisa nas classes de alunos aos participantes, sendo que efetuaram a leitura e assinaram o TCLE - Termo de Consentimento Livre e Esclarecido.

Buscou-se identificar a correlação entre Habilidades Sociais e desempenho acadêmico, bem como comparar resultados encontrados entre os cursos da área da saúde e os cursos de ciências exatas e sociais aplicadas, valendo-se do instrumento Statistical Package for the Social Sciences (IBM SPSS) 21.0 para Windows.

\section{Local}

O estudo foi realizado em uma universidade privada localizada na cidade de Francisco Beltrão, no estado do Paraná - Brasil. A universidade oferece ensino na modalidade presencial, semipresencial e ead em diversos cursos das áreas da saúde, humanas, sociais aplicadas e exatas, englobando cerca de três mil e quinhentos alunos pertencentes à região sudoeste.

\section{Participantes}

Conforme apresentado na Tabela 1 participaram deste estudo 628 estudantes universitários, $66,56 \%(\mathrm{n}=418)$ são do gênero feminino, 33,12 \% $(\mathrm{n}=208)$ do gênero masculino e $0,32 \%(n=2)$ não informaram, com idades variando entre 18 e 55 anos, matriculados nas séries intermediárias ( $2^{\circ}$ e $3^{\circ}$ anos $)$ dos cursos da área da saúde, humanas, ciências exatas e sociais aplicadas. Destes, 55,25\% $(n=347)$ são estudantes dos cursos 
da área da saúde e humanas: Psicologia, Odontologia, Enfermagem, Nutrição; Educação Física e Farmácia; e 44,75\% $(n=281)$ são alunos dos cursos de ciências exatas e sociais aplicadas: Administração, Arquitetura e Urbanismo, Direito, Engenharia Civil e Ciências Contábeis.

Do público participante da pesquisa dos cursos da área da saúde, 54,75\% (n=190) trabalham; nos cursos de ciências exatas e sociais aplicadas, o número é expressamente maior, sendo que 84,34\% $(\mathrm{n}=237)$ trabalham. Do total de indivíduos, 50,63\% $(\mathrm{n}=318)$ moram com os pais; $47,75 \%(n=298)$ possuem financiamento estudantil ou bolsa, sendo 23,15\% $(n=69)$ bolsistas do PROUNI. Outros 66,78\% $(n=199)$ participam do FIES, $10,07 \%(n=30)$ possuem outro tipo de financiamento para custear os estudos.

Tabela 1

Descrição dos participantes por curso e gênero

\begin{tabular}{lcccc}
\hline \multirow{2}{*}{ Curso } & Participantes & \multicolumn{3}{c}{ Gênero } \\
\cline { 3 - 5 } & & Masculino & Feminino & Outros \\
\hline Engenharia Civil & 40 & 29 & 11 & 0 \\
Direito & 94 & 44 & 50 & 0 \\
Arquitetura & 67 & 24 & 43 & 0 \\
Ciências Contábeis & 31 & 11 & 20 & 0 \\
Administração & 49 & 28 & 21 & 0 \\
Psicologia & 93 & 14 & 79 & 0 \\
Educação Física & 39 & 16 & 23 & 0 \\
Enfermagem & 55 & 7 & 48 & 0 \\
Farmácia & 39 & 10 & 29 & 0 \\
Administração & 27 & 2 & 25 & 0 \\
Odontologia & 95 & 24 & 69 & 2 \\
\hline
\end{tabular}

\section{Instrumentos}

O instrumento utilizado para verificar o repertório de Habilidades Sociais presentes no público pesquisado foi o Inventário de Habilidades Sociais IHS-Del-Prette (Del Prette \& Del Prette, 2014) considerado um instrumento de autorrelato, composto por um caderno de aplicação contendo 38 afirmações que descrevem uma possível situação de interação social e a possível resposta do indivíduo; uma folha de respostas com escala tipo likert (variando de nunca ou raramente para sempre ou quase sempre), manual para aplicação e interpretação, crivo para correção e folha de apuração. A fim de minimizar a desejabilidade social, alguns itens têm o valor invertido (na aplicação do crivo).

É possível obter acesso por meio da apuração das respostas ao escores total e aos escores fatoriais, sendo que estes se apresentam como: F1 - Enfrentamento com risco, cujas respostas representam reações a situações em que o sujeito precisa afirmar, defender direitos e respeitar os direitos dos demais com risco de resposta indesejável; F2 - Autoafirmação na expressão de afeto positivo, este fator envolve baixo risco de resposta indesejável e corresponde à forma como o indivíduo lida com as situações onde 
necessita expressar afeto e autoestima; F3 - Conversação e desenvoltura social, este fator verifica como as pessoas lidam quando estão em situações consideradas neutras com poucas chances de reação indesejável, como iniciar ou encerrar conversas pedir favores ou recusar pedidos; F4 - Autoexposição a desconhecidos ou a situações novas, em que se exige, em situação social, a habilidade de abordar ou se apresentar, falar perante pessoas desconhecidas, havendo risco de reação indesejável; F5 -Autocontrole da agressividade, compreende ser capaz de lidar com situações aversivas, incluindo receber críticas, bem como saber discordar ou expressar desagrado de maneira assertiva (Del Prette \& Del Prette, 2014; Del Prette, Del Prette \& Barreto 1998; Carraro, Rodrigues \& Araujo, 2016; Lima, 2006).

Para a variável desempenho acadêmico, foi adotado o critério nota como medida, embora não seja esta a única e mais adequada forma de mensuração, é a que melhor se ajusta e se apresenta como possível para identificação da variável. Miranda et al. (2015) apresentam a problematização quanto à medida a ser adotada para verificar o desempenho acadêmico "O primeiro desafio que se apresenta a quem pretende analisar desempenho acadêmico é definir a forma de mensurá-lo", pois o desempenho acadêmico, segundo Mamede et al. (2015), resulta de vários fatores por isso se torna difícil estabelecer parâmetros para medi-lo. Assim, para este estudo será considerada a nota compatível ao primeiro bimestre. Os dados foram cedidos pela instituição, estando os alunos cientes e de acordo.

\section{Resultados}

Por ser uma amostra não paramétrica, os dados foram analisados por meio da correlação de Spearman, tendo sido realizada a correlação entre as variáveis de Habilidades Sociais e desempenho acadêmico curso a curso nas áreas da saúde, humanas, exatas e sociais aplicadas. Considerou-se o escore total do IHS-Del-Prette (Del Prette \& Del Prette, 2014) para Habilidades Sociais e a média das notas obtidas pelos participantes no período da coleta de dados para a variável desempenho acadêmico.

Conforme demonstrado na tabela 2, os resultados evidenciam que, nos cursos da área de saúde e humanas, as Habilidades Sociais e desempenho acadêmico correlacionamse. Em contrapartida, os cursos de exatas e sociais aplicadas não apresentam correlação estatisticamente significativa.

Tabela 2

Dados da correlação entre Habilidades Sociais e desempenho acadêmico

\begin{tabular}{|c|c|c|}
\hline Curso & $\begin{array}{l}\text { Correlação entre Habilidades } \\
\text { Sociais e desempenho } \\
\text { acadêmico }\end{array}$ & $\begin{array}{l}\text { Correlação entre os fatores específicos } \\
\text { do IHS - Del -Prette (Del Prette \& Del } \\
\text { Prette, 2014) e desempenho acadêmico. }\end{array}$ \\
\hline Engenharia Civil & $(\rho=0,004 ; \mathrm{p}=0,983)$ & $\begin{array}{c}\text { A correlação não foi estatisticamente } \\
\text { significativa. }\end{array}$ \\
\hline Direito & $(\rho=0,001 ; \mathrm{p}=0,995)$ & $\begin{array}{l}\text { A correlação não foi estatisticamente } \\
\text { significativa. }\end{array}$ \\
\hline
\end{tabular}




\begin{tabular}{|c|c|c|}
\hline Curso & $\begin{array}{l}\text { Correlação entre Habilidades } \\
\text { Sociais e desempenho } \\
\text { acadêmico }\end{array}$ & $\begin{array}{l}\text { Correlação entre os fatores específicos } \\
\text { do IHS - Del -Prette (Del Prette \& Del } \\
\text { Prette, 2014) e desempenho acadêmico. }\end{array}$ \\
\hline Arquitetura e Urbanismo & $(\rho=-, 007 ; \mathrm{p}=0,957)$ & $\begin{array}{c}\text { A correlação não foi estatisticamente } \\
\text { significativa. }\end{array}$ \\
\hline Ciências Contábeis & $(\rho=-, 052 ; \mathrm{p}=0,796)$ & $\begin{array}{c}\text { A correlação não foi estatisticamente } \\
\text { significativa. }\end{array}$ \\
\hline Administração & $(\rho=-, 205 ; \mathrm{p}=0,223)$ & F5 $\left(\rho=0,385 ; \mathrm{p}=0,011^{*}\right)$ \\
\hline Psicologia & $\left(\rho=0,410 ; \mathrm{p}=0,000^{* *}\right)$ & $\begin{array}{c}\text { F5 }\left(\rho=0,410 ; \mathrm{p}=0,000^{* *}\right) \\
\text { F3 }\left(\rho=0,371 ; \mathrm{p}=0,000^{* *}\right) \\
\text { F2 }\left(\rho=0,390 ; \mathrm{p}=0,000^{* *}\right) \\
\text { F1 }\left(\rho=0,410 ; \mathrm{p}=0,000^{* *}\right) \\
\text { F4 }\left(\rho=0,224 ; \mathrm{p}=0,031^{*}\right)\end{array}$ \\
\hline Educação Física & $\left(\rho=0,419 ; \mathrm{p}=0,009^{* *}\right)$ & $\begin{array}{l}\text { F1 }\left(\rho=0,419 ; \mathrm{p}=0,009^{* *}\right) \\
\text { F5 }\left(\rho=0,515 ; \mathrm{p}=0,001^{* *}\right)\end{array}$ \\
\hline Enfermagem & $(\rho=0,578 ; \mathrm{p}=0,000 * *)$ & $\begin{array}{l}\text { F1 }\left(\rho=0.578 ; \mathrm{p}=0,000^{* *}\right) \\
\text { F5 }\left(\rho=0,422 ; \mathrm{p}=0,001^{* *}\right) \\
\text { F3 }\left(\rho=0,339 ; \mathrm{p}=0,011^{*}\right)\end{array}$ \\
\hline Farmácia & $\left(\rho=0,322 ; \mathrm{p}=0,046^{*}\right)$ & $\begin{array}{l}\text { F4 }\left(\rho=0,430 ; \mathrm{p}=0,006^{* *}\right) \\
\text { F5 }(\rho=0,522 ; \mathrm{p}=0,001 * *) .\end{array}$ \\
\hline Nutrição & $(\rho=0,507 ; \mathrm{p}=0,007 * *)$ & $\begin{array}{l}\text { F1 }\left(\rho=0,507 ; \mathrm{p}=0,007^{* *}\right) \\
\text { F3 }\left(\rho=0,564 ; \mathrm{p}=0,002^{* *}\right) \\
\text { F5 }\left(\rho=0,521 ; \mathrm{p}=0,005^{* *}\right) \\
\text { F2 }\left(\rho=0,434 ; \mathrm{p}=0,024^{*}\right)\end{array}$ \\
\hline Odontologia & $\left(\rho=0,457 ; \mathrm{p}=0,000^{* *}\right)$ & $\begin{array}{l}\text { F1 }\left(\rho=0,457 ; \mathrm{p}=0,000^{* *}\right) ; \\
\text { F2 }\left(\rho=0,352 ; \mathrm{p}=0,000^{* *}\right) ; \\
\text { F3 }\left(\rho=0,278 ; \mathrm{p}=0,006^{* *}\right) ; \\
\text { F5 }\left(\rho=0,349 ; \mathrm{p}=0,001^{* *}\right) ; \\
\text { F4 }\left(\rho=0,258 ; \mathrm{p}=0,012^{*}\right)\end{array}$ \\
\hline
\end{tabular}

Nota. $* * \mathrm{p}<0,01 ;{ }^{*} \mathrm{p}<0,05 ; \mathrm{F} 1$-Enfrentamento com risco; F2-Autoafirmação na expressão de afeto positivo; F3- Conversação e desenvoltura social; F4-Autoexposição a desconhecidos ou situações novas; F5-Autocontrole da agressividade.

Ao serem analisados os dados, não foram identificadas correlações estatisticamente significativas entre as variáveis para os cursos de Engenharia Civil ( $\rho=$ $0,004 ; \mathrm{p}=0,983)$, Direito $(\rho=0,001 ; \mathrm{p}=0,995)$, Arquitetura e Urbanismo $(\rho=-0,007$; $\mathrm{p}=0,957)$, Ciências Contábeis $(\rho=-, 052 ; \mathrm{p}=0,796)$, Administração $(\rho=0-, 205 ; \mathrm{p}=$ 0,223 ). Em contrapartida, foram identificadas correlações para os cursos de Psicologia $\left(\rho=0,410 ; \mathrm{p}=0,000^{* *}\right)$, Educação Física $(\rho=0,419 ; \mathrm{p}=0,009 * *)$, Enfermagem $(\rho=$ 0,$\left.578 ; \mathrm{p}=0,000^{* *}\right)$, Farmácia $\left(\rho=0,322 ; \mathrm{p}=0,046^{*}\right)$, Nutrição $\left(\rho=0,507 ; \mathrm{p}=0,007^{* *}\right)$ e Odontologia $\left(\rho=0,457 ; \mathrm{p}=0,000^{* *}\right)$.

Quando analisadas a correlação entre desempenho acadêmico e os fatores específicos do IHS-Del-Prette (Del Prette \& Del Prette, 2014), o curso de Psicologia apresentou correlações significativas para F5 $\left(\rho=0,410 ; \mathrm{p}=0,000^{* *}\right), \mathrm{F} 3\left(\rho=0,371 ; \mathrm{p}=0,000^{* *}\right)$, F2 $\left(\rho=0,390 ; \mathrm{p}=0,000^{* *}\right), \mathrm{F} 1\left(\rho=0,410 ; \mathrm{p}=0,000^{* *}\right)$ e correlação marginalmente significativa F4 $\left(\rho=0,224 ; \mathrm{p}=0,031^{*}\right)$. Para o curso de Educação Física, identificou-se 
correlação significativa entre F1 $(\rho=0,419 ; \mathrm{p}=0,009 * *)$ e F5 $(\rho=0,515 ; \mathrm{p}=0,001 * *)$ sem correlação estatisticamente significativa para os outros fatores. No curso de Enfermagem, F1 $\left(\rho=0,578 ; \mathrm{p}=0,000^{* *}\right)$ e F5 $(\rho=0,422 ; \mathrm{p}=0,001 * *)$ correlacionaram-se significativamente e F3 $\left(\rho=0,339 ; \mathrm{p}=0,011^{*}\right)$ correlacionou-se marginalmente. Houve correlação significativa entre dois dos fatores de escores do IHS-Del-Prette (Del Prette \& Del Prette, 2014) e desempenho acadêmico no curso de Farmácia F5 ( $\rho=0,522 ; \mathrm{p}=0,001$; **); F4 $\left(\rho=0,430 ; \mathrm{p}=0,006^{* *}\right)$. Para o curso de Nutrição, os resultados apresentaram correlação significativa para F1 $(\rho=0,507 ; \mathrm{p}=0,007 * *), \mathrm{F} 3(\rho=0,564 ; \mathrm{p}=0,002 * *), \mathrm{F} 5$ $\left(\rho=0,521 ; \mathrm{p}=0,005^{* *}\right)$, e marginalmente significativa para F2 $\left(\rho=0,434 ; \mathrm{p}=0,024^{*}\right)$. $\mathrm{O}$ curso de Odontologia apresentou correlações significativa para $\mathrm{F} 1(\rho=0,457 ; \mathrm{p}=$ $\left.0,000^{* *}\right), \mathrm{F} 2\left(\rho=0,352 ; \mathrm{p}=0,000^{* *}\right), \mathrm{F} 3\left(\rho=0,278 ; \mathrm{p}=0,006^{* *}\right), \mathrm{F} 5(\rho=0,349 ; \mathrm{p}=$ $0,001 * *)$ e marginalmente significativa para F4 $\left(\rho=0,258 ; \mathrm{p}=0,012^{*}\right)$.

O curso de Administração foi o único curso da área das ciências exatas e sociais aplicadas no qual foi possível identificar correlação entre desempenho acadêmico e os fatores de escores específicos do IHS-Del-Prette (Del Prette \& Del Prette, 2014) F5, Autocontrole da agressividade e assertividade $\left(\rho=0,385 ; \mathrm{p}=0,011^{*}\right)$.

Percebe-se, por meio dos resultados apresentados, que o fator que mais se correlacionou com desempenho acadêmico foi F5 Autocontrole da agressividade e assertividade, em que houve seis correlações significativas e uma correlação marginalmente significativa, seguido por F1 Enfrentamento com risco, que se correlacionou significativamente ao desempenho acadêmico em cinco cursos; Enquanto F3, Conversação e desenvoltura social, apresentou três correlações significativas e uma marginalmente significativa.

Para F2, Autoafirmação na expressão de afeto e sentimento positivo, houve duas correlações significativas e uma marginalmente significativa; e, finalmente, F4 Autoexposição a desconhecidos e situações novas foi o fator que menos correlacionou com desempenho acadêmico, uma correlação significativa e duas marginalmente significativas.

\section{Discussão}

Pelos resultados apresentados anteriormente, pode-se perceber que o desempenho acadêmico apresenta correlação direta com as Habilidades Sociais nos cursos da área da saúde e humanas, Psicologia $\left(\rho=0,410 ; \mathrm{p}=0,000^{* *}\right)$; Educação Física ( $\rho$ $=0,419 ; \mathrm{p}=0,009 * *)$; Enfermagem $(\rho=0,578 ; \mathrm{p}=0,000 * *)$; Farmácia $(\rho=0,322 ; \mathrm{p}$ $\left.=0,046^{*}\right)$; Nutrição $(\rho=0,507 ; \mathrm{p}=0,007 * *)$; Odontologia $(\rho=0,457 ; \mathrm{p}=0,000 * *)$. Resultado semelhante encontra-se no estudo de Gomes \& Soares (2013), o qual buscou verificar a correlação entre Habilidades Sociais, inteligência, expectativas acadêmicas e desempenho em universitários, no qual se constatou que Habilidades Sociais influenciam no desempenho acadêmico. Iturra, Goic, Astete e Jara (2012) respaldam, por meio dos seus resultados, os achados do presente estudo, ao verificarem que Habilidades Sociais se associam ao rendimento da academia, soma-se a pesquisa de Feitosa, Matos, Del Prette e Del Prette (2009) na qual se constata que rendimento escolar e comportamentos habilidosos se relacionam, especialmente os assertivos. 
As Habilidades Sociais influenciam o desempenho acadêmico por meio das inter-relações sociais e atuam como fatores de proteção pela melhora da convivência do universitário com professores, colegas e funcionários da instituição. "Considerando que os alunos utilizam suas habilidades pessoais com o objetivo de atingirem melhor desempenho acadêmico e para se motivarem a dar continuidade no curso escolhido [...]" (Gomes \& Soares, 2013) estes se tornam facilitadores dos processos de adaptação e aquisição de conhecimentos (Del Prette \& Del Prette, 2003). Soares, Mourão, Santos e Mello (2015) concordam com esta afirmação "[...] o nível de habilidades sociais poderia favorecer a integração dos estudantes com o mundo acadêmico, modificando a percepção dos mesmos sobre suas vivências acadêmicas [...]".

Faz-se importante ressaltar que o estudo de Iturra et al. (2012) verifica que a correlação entre Habilidades Sociais e desempenho é presente especialmente nos resultados do público feminino, tornando-se significativa a colaboração dos resultados desse estudo com os apresentados nessa pesquisa, já que 78,67\% da amostra dos cursos de saúde e humanas pertencem ao gênero feminino. Os autores referem-se à capacidade dos indivíduos de gênero feminino tirarem maior proveito da qualidade dos seus relacionamentos interpessoais, implicações quanto à cooperação e colaboração que melhoram seu rendimento acadêmico.

Entre os cursos da área de Exatas e Sociais Aplicadas, as variáveis desempenho acadêmico e Habilidades Sociais não apresentaram correlação estatisticamente significativa: Engenharia Civil $(\rho=0,004 ; \mathrm{p}=0,983)$; Direito $(\rho=0,001 ; \mathrm{p}=0,995)$; Arquitetura e Urbanismo $(\rho=-, 007 ; \mathrm{p}=0,957)$; Ciências Contábeis $(\rho=-, 052 ; \mathrm{p}=$ $0,796)$; Administração $(\rho=-, 205 ; \mathrm{p}=0,223)$. Del Arco (2005) expressa em seu estudo a não correlação entre Habilidades Sociais e desempenho acadêmico, corroborando os resultados aqui apresentados.

Os resultados deste estudo confirmam os dados encontrados no estudo de BolsoniSilva e Loureiro (2015), em que os autores afirmam que, no contexto universitário, estudantes das áreas de saúde e humanas são mais habilidosos, segundo as médias obtidas, do que os estudantes matriculados nos cursos de ciências exatas.

Ao serem analisados os fatores específicos dos escores do IHS-Del-Prette (Del Prette \& Del Prette, 2014) que contemplam classes de respostas pertencentes a categorias de Habilidades Sociais (Del Prette \& Del Prette, 2014), verificou-se a correlação entre estes e desempenho acadêmico, para o fator F1 - Enfrentamento com risco, houve a ocorrência em cinco (5) cursos - Psicologia, Educação Física, Enfermagem, Nutrição e Odontologia.

No estudo de Carneiro e Teixeira (2011), os autores ressaltam que a maior parte dos sujeitos participantes da sua pesquisa apresentaram um repertório elaborado em Habilidades Sociais neste fator, que segundo Del Prette et al. (1998) está relacionado à assertividade. Soares et al. (2009) apontam que, quanto maior o déficit em enfrentamento com risco, pior é o relacionamento com os colegas.

Nos cursos de Psicologia, Nutrição, Odontologia o fator F2, autoafirmação na expressão de afeto positivo, correlacionou-se com desempenho acadêmico. De acordo com Del Prette et al. (1998), este fator retrata "demandas interpessoais de expressão de afeto positivo e de afirmação da autoestima, com risco mínimo de reação indesejável". 
Gomes e Soares (2013) encontraram correlação entre este fator e o bom rendimento acadêmico. Carneiro e Teixeira (2011) apresentam, em sua pesquisa, este fator como o único onde os alunos mais adiantados no curso são mais habilidosos que os iniciantes.

Assim, alunos com dificuldades nestas classes de comportamento tendem a aprimorar seu desempenho social ao participarem de atividades que exigem essa resposta no meio acadêmico. Bolsoni-Silva e Loureiro (2015) afirmam que as mulheres tendem a apresentarem maiores médias neste fator. Temos ainda, a contribuição de Soares et al. (2009):

[...] quanto maior é a expressão de afeto positivo maior é a aquisição do conhecimento para o curso, mais eficientes são os métodos de estudo, o desenvolvimento da carreira, o relacionamento com os professores, a adaptação do curso e a gestão do tempo. O envolvimento afetivo traz beneficios para a relação acadêmica. (Soares et al., 2009. p. 37).

As características do F3, Conversação e desenvoltura social, conforme Del Prette et al. (1998), "retratam situações neutras (em termos de afeto positivo ou negativo) de aproximação com risco mínimo de reação indesejada, demandando principalmente, 'traquejo social' na conversação o que supõem conhecimento das normas de relacionamento". Para Carneiro e Teixeira (2011), os resultados demonstram que este foi o fator com as maiores médias. Na presente pesquisa, o F3 correlacionou-se ao desempenho acadêmico nos cursos de Psicologia, Nutrição, Odontologia e Enfermagem. Soares et al. (2009) reconhecem que os comportamentos que compõem este fator refletem no bem estar físico e psicológico, melhorando a percepção de competência.

Bolsoni-Silva e Loureiro (2015), por sua vez, revelam a importância desses aspectos para o universitário: "Na vida universitária, um repertório de habilidades interpessoais e de desempenho de falar em público pode ser considerado imprescindível para um melhor desempenho acadêmico e social dos indivíduos".

Ao quarto fator, F4 Autoexposição a desconhecidos e situações novas, atribui-se as características relacionadas à realização de perguntas, inquerir auxílio e favores a desconhecidos (Carneiro \& Teixeira, 2011). Este aparece correlacionado ao desempenho acadêmico no curso de Psicologia e Farmácia no presente estudo, ainda assim, é o fator que menos se correlaciona. Os achados convergem com o estudo de Del Prette e Del Prette (2003) no qual o fator F4 aponta para a necessidade de intervenções por correlacionarse menos que os outros fatores e por ser constituído por itens que são importantes no contexto profissional.

O fator F5 Autocontrole de agressividade e assertividade aparece correlacionado a sete dos cursos pesquisados; seis da área da saúde: Psicologia, Nutrição, Odontologia, Enfermagem, Educação Física e Farmácia, e ainda, ao curso de Administração, da área de ciências exatas e sociais aplicadas. O estudo de Gomes e Soares (2013) aponta resultados no qual o fator F5 relacionou-se a médias maiores de desempenho acadêmico, ou seja, quanto mais habilidoso o universitário for nestes repertórios que se relacionam a assertividade, melhor é o resultado do seu rendimento acadêmico. 
Estes resultados contrariam o que Brandão (2016) postula, tendo em vista que no seu estudo o fator F5, Autocontrole da agressividade, não interfere no bom desempenho acadêmico, o que sugere a importância da realização de mais pesquisas referentes à assertividade de desempenho acadêmico.

Os dados do estudo de Carneiro e Teixeira (2011) evidenciaram, no público pesquisado, bons índices neste fator, sendo possível se pensar em duas hipóteses: que a maior parte dos alunos reage de maneira adequada à estimulação aversiva ou esquivam-se de situações nas quais essas condutas se revelam, podendo ser, dessa forma, considerados agressivos pelos pares.

As informações elencadas do presente estudo evidenciam que existe uma diferença quanto à correlação entre Habilidades Sociais e desempenho acadêmico entre os cursos de ciências exatas e sociais aplicadas e da área da saúde e humanas. Isto pode se dar em decorrência da ênfase no ensino de habilidades técnicas em detrimento das Habilidades Sociais nos cursos de ciências exatas e sociais aplicadas.

Lopes, Dascanio, Ferreira, Del Prette e Del Prette (2017, p. 57) problematizam essa questão ao afirmarem que: "Na formação universitária, essa carência pode ser ainda mais crítica nas áreas de exatas e tecnológicas por tradicionalmente, não contemplarem esses temas, mais comuns nas ciências humanas". Em contrapartida, convergem os resultados nos cursos de saúde e humanas, o que tenta ser explicado levando-se em conta que esses cursos compõem-se por público maioritariamente feminino, corroborando com o estudo de Iturra et al. (2012), anteriormente citado. Bolsoni-Silva e Loureiro (2015) sugerem que essas habilidades são mais estimuladas pela realização de trabalhos em grupo e apresentação de seminários, as quais podem ser efetivas ao permitirem o treino desses comportamentos durante a graduação.

\section{Considerações finais}

O presente estudo, enquanto produção científica, contribui para o avanço da construção do conhecimento em Psicologia, por meio do levantamento de dados empíricos e análise de variáveis relacionadas a temas de interesse desta ciência, como os aqui considerados (Habilidades Sociais e desempenho acadêmico). Ao se propor a investigação destes temas, lança-se a visibilidade sobre aspectos importantes para o desenvolvimento de estratégias voltadas a estas questões e ao público.

Como foi apresentado, as Habilidades Sociais envolvem fatores psicológicos e comportamentais arrolados pelo instrumento utilizado IHS-Del-Prettte (Del Prette \& Del Prette, 2014) como, F1 Enfrentamento com risco; F2 Autoafirmação na expressão de afeto positivo; F3 Conversação e desenvoltura social; F4 Autoexposição a desconhecidos e situações novas; F5 Autocontrole de agressividade e assertividade; e são, segundo Gomes e Soares (2013), influenciadores do desempenho acadêmico pelo fato de este ser multideterminado (Rocha et al., 2018).

$\mathrm{Na}$ pesquisa realizada, essas duas variáveis (Habilidades sociais e desempenho acadêmico) apareceram correlacionadas nos cursos de saúde e humanas, o que corrobora com os achados teóricos. Em contrapartida, os cursos de ciências exatas e sociais aplicadas 
não apresentaram correlação estatisticamente significativa, o que se evidencia no resultado da pesquisa de Del Arco (2005), o qual identificou não haver correlação entre desempenho acadêmico e Habilidades Sociais para este público.

Neste sentido os resultados indicam que os cursos dessa área podem ser alvo de treinamento em Habilidades Sociais, sendo válido ressaltar que essas são questões importantes para todos, independentemente da área do conhecimento, diante das habilidades e competências necessárias para a formação e a atuação profissional futura.

Durante a realização deste estudo foi possível elencar a escassez de literatura que abarque as variáveis pesquisadas, tendo em vista que encontra-se na literatura produções que se propõem a investigar tanto o desempenho acadêmico quanto Habilidades Sociais, porém poucos procuram correlacioná-los, o que existe até o momento, são produções que priorizam a questão de variáveis sociodemográficas em detrimento de pesquisas que levantem dados sobre variáveis comportamentais e características psicológicas o que justifica a intenção e a relevância da pesquisa realizada.

Além disso, o método de seleção da amostra (conveniência) pode ser considerado um limitador, caberia neste campo, investigar como estas variáveis influenciam-se mutuamente em públicos de contextos universitários diversos, como os de universidades públicas ou de outras regiões do país.

Resgata-se que, neste sentido, cabe também investigar-se o porquê das diferenças dos resultados entre as áreas do conhecimento pesquisadas. O que difere (ambiente, comportamento, contingências) nos estudantes dos cursos das áreas da saúde e humanas dos estudantes das áreas de ciências exatas e sociais aplicadas? Por que no primeiro grupo as variáveis correlacionam-se, e no segundo não há correlação? Não estariam os estudantes priorizando o desempenho às Habilidades Sociais por considerarem esse mais relevante? Levantam-se algumas hipóteses, sancionadas por outros estudos, como o fato de o número de indivíduos do gênero feminino ser maior nos cursos da área de saúde e humanas em comparação aos dos cursos de ciências exatas e sociais aplicadas. Mas, o que há além disso? Lança-se o questionamento a quem se propor a discutir o tema e as questões abordadas.

Diante disso, tornam-se importantes posteriores estudos a fim de entender e ampliar os resultados apresentados, bem como a possibilidade da elaboração de estratégias que favoreçam a aquisição e a ampliação do repertório habilidoso no público universitário, considerando a importância e a contribuição das Habilidades Sociais ao contexto acadêmico, independente da área do conhecimento para o desenvolvimento de habilidades e competências para atuação profissional futura.

\section{Referências}

Bolsoni-Silva, A. T., \& Carrara, K. (2010). Habilidades sociais e análises do comportamento compatibilidades e dissenções conceitual-metodológicas. Psicologia em revista, 16(2), 330-350.

Bolsoni-Silva, A. T., \& Loureiro, S. R. (2015). Social skills of undergraduates without mental disorders: Academic and socio-demographic variables. Psico-USF, 20(3), 447-459. 
Brandão, A. S. (2016). Desempenho acadêmico de universitários, variáveis preditoras: habilidades sociais, saúde mental, características sociodemográficas e escolares. Tese de Doutorado, Programa de Pós-Graduação em Psicologia, Universidade de São Paulo. São Paulo, SP.

Caballo, V. E. (1955/2016). Manual de avaliação e treinamento das habilidades sociais. São Paulo: Santos.

Carneiro, A. A., \& Teixeira, C. M. (2011.) Avaliação de Habilidades Sociais em Alunos de Graduação em psicologia da universidade federal do maranhão. Psicologia: ensino e formação, 2(1), 43-56.

Carraro, C. R., Rodrigues, M. S., \& Araujo, E. A. S. (2016). Habilidades Sociais profissionais presentes em engenheiros alunos de MBA de uma universidade no interior de São Paulo. Latin American Journal of Business Management, 7(2), 298312 .

Cozby. P. C. (2012). Métodos de Pesquisa em ciências do comportamento. São Paulo: Atlas.

Del Prette, A., \& Del Prette, Z. A. P. (2011). Psicologia das relações interpessoais: vivências para o trabalho em grupo. Petrópolis: Vozes.

Del Prette, A., \& Del Prette, Z. A. P. (2017). Habilidades Sociais: intervenções efetivas em grupo. São Paulo: Pearson Clinical Brasil.

Del Prette, Z. A. P., \& Del Prette, A. (2003). No contexto da travessia para o ambiente de trabalho: treinamento de habilidades sociais com universitários. Estudos de Psicologia, 8(3), 413-420.

Del Prette, Z. A. P., \& Del Prette, A. (2014). Inventário de habilidades sociais (IHS -Del Prette): Manual de aplicação, apuração e interpretação. São Paulo: Casa do Psicólogo.

Del Prette, Z. A. P., Del Prette, A., \& Barreto, M. C. M. (1998). Análise de um Inventário de Habilidades Sociais (IHS) em uma amostra de Universitários. Psicologia: Teoria e Pesquisa, 14(3), 219-228.

Del Arco, C. R. G. N. (2005). Habilidades Sociales, clima social familiar y rendimento académico en estudiantes universitários. Liberabit, 11(11), 63-74.

Elias, L. C. S., \& Amaral, M. V. (2016). Habilidades Sociais, Comportamentos e Desempenho Acadêmico em Escolares antes e após Intervenção. Psico-USF, 21(1), 49-61.

Fagundes, C. V., Luce, M. B., \& Espinar, S. R. (2014). O desempenho acadêmico como indicador de qualidade da transição Ensino Médio-Educação Superior. Ensaio: Avaliação e Políticas Públicas em Educação, 22(84), 635-670.

Feitosa, F. B., Matos, M. G., Del Prette, Z. A. P., \& Del Prette, A. (2009). Desempenho acadêmico e interpessoal em adolescentes portugueses. Psicologia em Estudo, 14(2), 259-266.

Ferreira, V. S., Oliveira, M. A., \& Vandenberghe, L. (2014). Efeitos a Curto e Longo Prazo de um Grupo de Desenvolvimento de Habilidades Sociais para Universitários. Psicologia: Teoria e Pesquisa, 30(1), 73-81. 
Gomes, G., \& Soares, A. B. (2013). Inteligência, Habilidades Sociais e Expectativas Acadêmicas no Desempenho de Estudantes Universitários. Psicologia: Reflexão e crítica, 26(4), 780-789

Iturra, G. O., Goic, C. E., Astete, E. P., \& Jara, M. O. (2012). Habilidades Sociales y Rendimiento Académico: Uma Mirada desde el género. Acta Colombiana de Psicología, 15(2), 21-28.

Junior, L. H. S., \& Amorim, J. G. (2014). Fatores socioeconômicos que influenciam o desempenho educacional: Uma análise dos alunos concluintes da autarquia educacional de Belo Jardim no agreste de Pernambuco. Economia e Desenvolvimento, 13(1), 168-201.

Lima, T. H. (2006). Inventário de Habilidades Sociais: Pioneirismo na avaliação clínica, educacional e organizacional. Avaliação Psicológica, 5(2), 277-278.

Lopes, D. C., Dascanio, D., Ferreira, B. C., Del Prette, Z. A. P., \& Del Prette, A. (2017). Treinamento de Habilidades Sociais: Avaliação de um programa de desenvolvimento interpessoal profissional para universitários de ciências exatas. Interações em Psicologia, 21(1), 55-65.

Mamede, S. P. N., Marques, A. V. C., Rogers, P., \& Miranda, G. J. (2015). Determinantes Psicológicos do Desempenho Acadêmico em Ciências Contábeis: Evidências do Brasil. Brazilian Business Review, 12, 54-75.

Miranda, G. J., Lemos, K. C. S., Oliveira, A. S., \& Ferreira, M. A. (2015). Determinantes do Desempenho Acadêmico na Área de Negócios. Revista Meta: Avaliação, 7(20), 175-209.

Moreira, M. B., \& Medeiros, C. A. (2007). Princípios básicos da análise do comportamento. Porto Alegre: Artmed.

Rocha, A. L. P., Leles, C. R., \& Queiroz, M. G. (2018). Fatores associados ao desempenho acadêmico de estudantes de Nutrição no Enade. Revista brasileira de Estudos pedagógicos, 99(251), 74-94.

Rodrigues, B. C. O., Resende, M. S., Miranda, G. J., \& Pereira, J. M. (2016). Determinantes do desempenho acadêmico dos alunos dos cursos de Ciências Contábeis no ensino à distância. Enfoque: Reflexão Contábil, 35(2), 139-153.

Santos, C. A., Cunha, H. C., \& Hein, N. (2017). Fatores relacionados ao desempenho dos acadêmicos dos cursos de ciências contábeis. Revista de Estilos de Aprendizagem. Orem-Utah-USA, 9(18), 67-91.

Silva, H. G. (2015). Fatores determinantes do desempenho acadêmico no ensino superior: Uma abordagem por meio do estado da arte. [Resumo]. Em: Programa de Pós-Graduação em Educação da Universidade de Uberaba (Org.), Resumos de comunicações científicas. III Congresso Internacional Trabalho Docente e Processos Educativos (pp. 1-15). Uberaba: UNIUBE.

Skinner, B. F. (1904/1972) Tecnologia do ensino. São Paulo: Editora da Universidade de São Paulo.

Soares, A. B., Buscacio, R. C. Z., Fernandes, A. M., Medeiros, H. C. P., \& Monteiro, M. C. (2017). O impacto dos comportamentos sociais acadêmicos nas habilidades sociais de estudantes. Revista Interinstitucional de Psicologia, 10(1), 69-80. 
Soares, A. B., Poubel, L. N., \& Mello, T. V. S. (2009). Habilidades sociais e adaptação acadêmica: um estudo comparativo em instituições de ensino público e privado. Aletheia, 29, 27-42.

Soares, A. B., Mourão, L., Santos, A. A. A. dos, \& Mello, T. V. dos S. (2015). Habilidades sociais e vivência acadêmica de estudantes universitários. Interação em Psicologia. Curitiba. 19(2), 211-223.

Thaís Cristina Gutstein Nazar: Doutorado em Educação pela Universidade Federal do Paraná (UFPR). Professora no Curso de Psicologia da Universidade Paranaense - UNIPAR. Av. Julio Assis Cavalheiro, 2000 Bairro Industrial CEP 85601-000. Francisco Beltrão/PR. Brasil. Telefone: (46) 991149438.

Michele Quaglioto Tartari: Acadêmica do curso de Psicologia da Universidade Paranaense - UNIPAR. Ana Caroline Grassi Vanazi: Acadêmica do curso de Psicologia da Universidade Paranaense - UNIPAR. Andreia Belusso: Acadêmica do curso de Psicologia da Universidade Paranaense- UNIPAR.

E-mail para contato: thaiscg@prof.unipar.br 